East African Medical Journal Vol. 86 No. 2 February 2009

GESTATIONAL THYROTOXICOSIS ASSOCIATED WITH EMESIS IN EARLY PREGNANCY

J. R. Ndungu, MBChB, MMed, Lecturer, A. Amayo, MBChB, MMed, Senior Lecturer, Department of Human Pathology, Z.P. Qureshi, MBChB, MMed, Senior Lecturer, Department of Obstetrics and Gynaecology and C. Sekadde Kigondu, BSc, PhD, Associate Professor, Department of Human Pathology, College of Health Sciences, University of Nairobi, P. O. Box 19676 - 00202, Nairobi, Kenya,

Request for reprints to: Dr. J.R. Ndungu, Department of Human Pathogy, College of Health Sciences, University of Nairobi, P. O. Box 19676 - 00202, Nairobi, Kenya

\title{
GESTATIONAL THYROTOXICOSIS ASSOCIATED WITH EMESIS IN EARLY PREGNANCY
}

\author{
J. R. NDUNGU, A. AMAYO, Z.P. QURESHI and C. SEKADDE KIGONDU
}

\begin{abstract}
Objectives: To determine the thyroid profile and the prevalence of gestational thyrotoxicosis among women with emesis during early pregnancy.

Design: A descriptive cross - sectional study.

Setting: Kenyatta National Hospital acute gynaecology ward and the ante-natal clinic.

Subjects: Seventy two women presenting with emesis up to 16 weeks gestation. Main outcome measures: The levels of FT3, FT4, TSH and $\beta$-hCG during the first 16 weeks of gestation. Correlation between the thyroid hormones and $\beta$-hCG as well as the severity of vomiting was also done.

Results: The point prevalence of gestational thyrotoxicosis was $8.3 \%$. There was a significant positive correlation between $\beta$-hCG levels and FT3 and FT4 (P-values $<0.05)$, and a significant negative correlation between $\beta$-hCG and TSH $(P<0.05)$. Correlation between the severity of vomiting and the thyroid hormones as well as $\beta$-hCG was not statistically significant. Patients' age ranged from 14-38 years (median 26). Majority of the women studied were at a gestation of 8 to 11 weeks $(38.9 \%)$. Most patients $(84.7 \%)$ had one to five episodes of vomiting per day. Peak $\beta$-hCG was at 12 - 15 weeks gestation.

Conclusions: Thyrotoxicosis does occur among women with emesis in pregnancy in this set-up. Screening for it may be beneficial to such women and also those with high serum $\beta$-hCG levels above the median for the gestational age.
\end{abstract}

\section{INTRODUCTION}

Maternal hyperthyroidism may have deleterious effects on the outcome of pregnancy. The most common cause of hyperthyroidism in women of child-bearing age (both pregnant and non-pregnant women) is Graves' disease. In recent years, another cause that has been characterised results from direct stimulatory effects of $\beta$-hCG on the thyroid. This can induce thyrotoxicosis transiently during the first half of gestation, and its prevalence in pregnancy much higher than that of Graves' disease, although it has usually less severe clinical manifestations. The syndrome, referred to as gestational transient thyrotoxicosis (GTT), differs from Graves' disease in that it is not of autoimmune origin, and the course, foetal risks, and management and follow-up of both entities are different (1).
The precise pathogenicmechanisms underlying GTT are still not fully understood. It is thought that abnormal molecular variants of $\beta$-hCG, with a prolonged half life, are produced in these situations explaining sustained high circulating $\beta$-hCG levels, or $\beta$-hCG variants with a more potent thyrotropic activity (2). It has also been hypothesised that a dysregulation of $\beta$-hCG production may transiently take place (3). A quantitative direct effect of elevated $\beta$-hCG to stimulate the thyroid gland may presumably be sufficient to explain GTT in most pregnant women, provided that $\beta$-hCG values remain above $75,000-$ $100,000 \mathrm{U} / \mathrm{L}$ for a sufficient period of time. GTT is directly related to both the amplitude and duration of peak $\beta$-hCG. Whatever the final explanation, the effects of $\beta$-hCG to stimulate the thyroid gland can best be explained by the marked homology that exists between the $\beta$-hCG and TSH molecules, as 
well as between the LH/ $\beta$-hCG and TSH receptors $(4,5)$. Thus, GTT can be considered an example of an endocrine "spillover "syndrome, a concept based on the molecular mimicry between hormone ligands and their receptors (5-7).

An unresolved question is whether the thyroid gland is the passive bystander (or the victim) of abnormal thyrotropic activity of $\beta$-hCG in GTT, or whether the gland itself, through variable degrees of sensitivity of the TSH receptor, may play an active role in its responsiveness to the action of $\beta$-hCG. So far, only one example has been reported with a substantially increased sensitivity of the TSH receptor to the stimulatory effect of $\beta-h C G$, due to a single mutation in the extracellular domain of the TSH receptor (KI83R) (8). The mutant TSH receptor was more sensitive than the wild-type receptor to $\beta$-hCG, thus accounting for recurrent thyrotoxicosis during pregnancy in the presence of normal $\beta$-hCG levels. This finding raises the possibility that some women who develop GTT may have an abnormality at the level of the thyroid follicular cell.

GTT is often associated with nausea (morning sickness), increased vomiting and hyperemesis gravidarum, a severe condition requiring hospitalisation and drastic treatment (9). Studies have established the correlation between severity of emesis and abnormalities of thyroid function.

\section{MATERIALS AND METHODS}

This was a prospective study conducted at Kenyatta National Hospital, Nairobi. Serum samples were obtained from 72 women presenting with emesis up to 16 weeks gestation. Permission was obtained from the $\mathrm{KNH}$ ethical committee and informed written consent was obtained from the participants. Patients who were known to have thyroid disorders before pregnancy and those with features of other underlying diseases were excluded.

Serum TSH, FT3, FT4 and $\beta$-hCG were estimated by immunoassay on an Abbott IMX machine. Samples were run in batches and three levels of controls (low, medium and high) were included in each run to validate the results. Validated manufacturer recommended reference values for T5H, FT3, FT4 and $\beta$-hCG were used to interpret the hormone values. Gestational thyrotoxicosis was defined as elevated FT4 and FT3 with lowTSH.

Data analysis was performed using SPSS (version 12.0). Logarithmic transformation was performed for $\beta$-hCG as it did not conform to a gaussian distribution. Correlations between continuous variables were assessed using Pearsons correlation coefficients. A P-value of $<0.05$ was considered statistically significant.

\section{RESULTS}

A total of 72 women with emesis during early pregnancy were studied. Their ages ranged from fourteen to thirty eight years with an average of twenty five years. The majority of the women were in the age group $24-28$ years. Parity ranged from $0+0$ to $4+4$.

Most of the patients $(84.7 \%)$ had moderate vomiting (one to five episodes of vomiting per day). The thyroid hormone levels were classified into low, normal or high based on the reference values. Normal thyroid profile values were found in most patients. There were six patients with elevated FT3 and /or FT4 levels with low TSH giving a point prevalence of gestational thyrotoxicosis of $8.3 \%$ in this study. Five (6.9\%) patients had high FT3levels alone. Four (5.6\%) of those with high FT3 levels also had high FT4 levels with two patients having either high FT3 levels or high FT4 levels. All those with high FT3 and / or high FT4 levels had low TSH levels. The two patients with low FT4 had normal TSH levels while one patient with a high TSH levels had a normal FT4/FT3 levels. One patient with low FT3 had accompanying low TSH while the other patient with low FT3 had normal TSH level. Nineteen patients (26.4\%) had low TSH levels (Table 1).

The $\beta$-hCG level among the 72 study subjects ranged from $1,664 \mathrm{miu} / \mathrm{ml}$ to $286,628 \mathrm{miu} / \mathrm{ml}$ with a mean of $121,740 \mathrm{miu} / \mathrm{ml}$. The peak $\beta$-hCG was at 12 -15 weeks gestation (mean 283,136 miu $/ \mathrm{ml}$ ) followed by a decline.

Table 1

Thyroid hormone levels in patients with emesis during pregnancy $(n=72)$

\begin{tabular}{lcc}
\hline Hormone level & No. & $(\%)$ \\
\hline FT3 $(\mathrm{pg} / \mathrm{dl})$ & & \\
Low & 2 & 2.8 \\
Normal & 65 & 90.3 \\
High & 5 & 6.9 \\
FT4 $(\mathrm{pg} / \mathrm{dl})$ & & \\
Low & 2 & 2.8 \\
Normal & 65 & 90.3 \\
High & 5 & 6.9 \\
TSH $(\mu \mathrm{iu} / \mathrm{ml})$ & & \\
Low & 19 & 26.4 \\
Normal & 52 & 72.2 \\
High & 1 & 1.4 \\
\hline
\end{tabular}

All those with high FT3 and / or FT4 levels had $\beta$-hCG levels above the mean value for the study subjects and also above the reference ranges for the gestation (Table 2). 
Table 2

TSH, $\beta-H C G$ and the gestation of the subjects with gestational thyrotoxicosis

\begin{tabular}{lcccc}
\hline FT3 $(\mathrm{pg} / \mathrm{ml})$ & FT4 $(\mathrm{ng} / \mathrm{dl})$ & TSH $(\mu \mathrm{iu} / \mathrm{ml})$ & $\beta$-HCG $(\mathrm{miu} / \mathrm{ml})$ & $\begin{array}{c}\text { Gestation } \\
\text { (Weeks) }\end{array}$ \\
\hline $1.68-3.54$ & $0.71-1.85$ & $0.47-5.0$ & $11,500-137,000$ & 10 \\
27.24 & 6.0 & 0.01 & 268,188 & 8 \\
3.74 & 1.77 & 0.13 & 274,808 & 11 \\
0.88 & 3.31 & 0.05 & 221,172 & 13 \\
22.06 & 5.90 & 0.00 & 174,400 & 16 \\
8.55 & 2.74 & 0.11 & 279,600 & 9 \\
4.66 & 2.35 & 0.16 & 142,560 & \\
\hline
\end{tabular}

There was a significant positive correlation between $\beta$-hCG levels and FT4 levels with a P-value of 0.025 . Those with high FT4 had corresponding higher levels of $\beta$-hCG compared to those with either low or normal FT4.

There was a significant positive correlation between $\beta$-hCG levels and FT3 levels with a P-value of 0.023 .

\section{Figure 1}

Correlation between the $\beta$-HCG levels and the TSH levels in patients with emesis

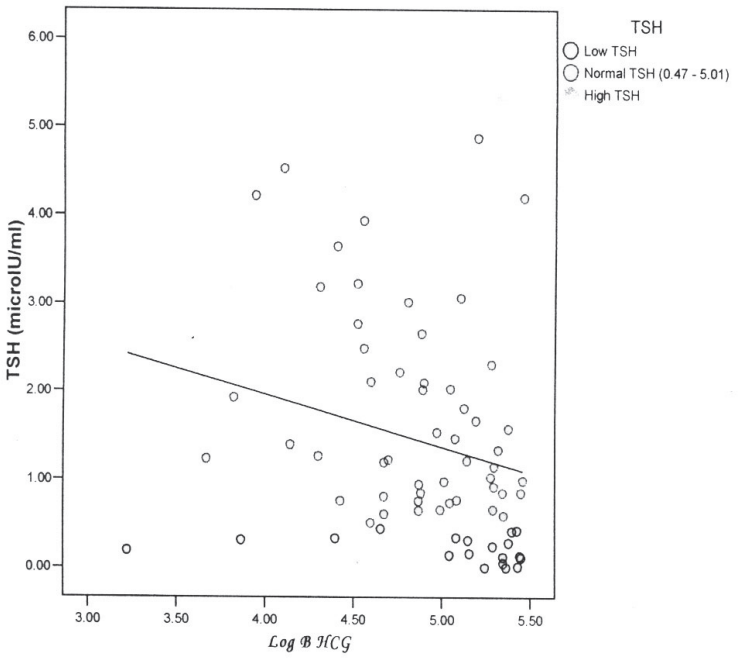

There was a significant negative correlation between $\beta$-hCG and the TSH levels ( $\mathrm{P}$-value $<0.05$ ) (Figure $1)$. The correlation between the severity of vomiting and the levels of FT3, FT4, TSH and $\beta$-hCG was not significant ( $p$-value $>0.05$ ).

\section{DISCUSSION}

The prevalence of gestational thyrotoxicosis in this study was found to be $8.3 \%$. Gestational thyrotoxicosis was defined as the presence of an elevated FT4 or FT3 or both with a corresponding depressed level of TSH in a patient with emesis during pregnancy. This prevalence of gestational thyrotoxicosis in this study corresponds with other studies done in other regions of the world where the prevalence of gestational thyrotoxicosis was found to vary from $0.3 \%$ in Japan to $11 \%$ in Hong Kong (6).

There were discordances between TSH and the free thyroid hormones in this study the most common being low TSH with normal FT3 and FT4 which was found in fourteen $(19.4 \%)$ patients. The possible explanations to this are sub-clinical thyrotoxicosis or resolving thyrotoxicosis. Subclinical thyrotoxicosis is defined as a low serum thyrotrophin (TSH) concentration in a patient with normal serum free thyroxine (FT4) and free triiodothyronine (FT3) concentrations. The secretion of TSH may be suppressed even in the presence of normal serum thyroid hormone levels. This reflects the highly sensitive response that the pituitary gland mounts to minor changes in serum FT4 and FT3 concentrations (10).

Studies have revealed that, in some cases the serum TSH may remain partially suppressed for several weeks after the FT 4 and FT3 have reverted to normal in patients who had gestational thyrotoxicosis previously $(3,11,12)$. Other studies showed that the serum TSH might be transiently suppressed in 10$20 \%$ of euthyroid women at the time of peak $\beta$-hCG levels (11).

Three patients had either low FT3 or FT4 levels with normal TSH values. These could possibly be cases of euthyroid sick syndrome where systemic non thyroidal diseases e.g. severe vomiting during pregnancy, infections or even toxaemia during pregnancy affect the free thyroid hormones. In this study all the patients had emesis some of them severe emesis $(15.3 \%)$ and this could have had effects on the thyroid hormones. Euthyroid sick syndrome can be described as abnormal findings on thyroid function tests that occur in the setting of a non thyroidal illness without pre-existing hypothalamic-pituitary and thyroid gland dysfunction. After recovery from non thyroidal illness, these thyroid function test result abnormalities should be completely reversible. 
The one patient with high TSH levels had normal FT3 and FT4 levels. This could probably be cases of early primary hypothyroidism. Hypothyroidism occurs during pregnancy relatively frequently. A nation-wide US survey showed that $4.6 \%$ of the population aged 12 years old and older had hypothyroidism and that $4.3 \%$ of all women suffered from thyroid disease or were taking thyroid medication. Routine prenatal screening showed that $2.2 \%$ of pregnant women in their second trimester had thyroid stimulating hormone (TSH) levels at or above $6 \mu \mathrm{IU} / \mathrm{L}$ (12).

There was one patient who had low TSH with low free thyroid hormones. Despite the fact that this was considered to be a case of secondary hypothyroidism further evaluation of the other anterior pituitary hormones needed to be done to confirm pituitary gland pathology. In this study a significant positive correlation was found between $\beta$-hCG and FT4/FT3 levels (P-values 0.025 and 0.023 for FT4 and FT3 respectively). A significant negative correlation between TSH and $\beta$-hCG levels was found ( $\mathrm{p}$-value 0.025). These findings are similar to those of Yeo et al (13) who investigated the prevalence of gestational thyrotoxicosis in Asian women in Singapore and found-out that the total and free $\beta$-hCG correlated negatively with TSH $(\mathrm{P}<0.0001$ and $\mathrm{P}<0.0001$, respectively), positively with FT4 $(\mathrm{P}<0.001$ and $\mathrm{P}<$ $0.001)$ and FT3 $(\mathrm{P}<0.001$ and $\mathrm{P}<0.01)$.

Similarly the positive correlation between the thyroid hormones and the $\beta$-hCG levels during pregnancy in this study corresponds with other studies which have shown that normal women may develop gestational transient thyrotoxicosis when they have abnormally elevated peak $\beta$-hCG levels and when these are sustained during an unusually prolonged period $(4,12)$.

Some reports have indicated that up to two thirds of pregnant women presenting with hyperemesis have biochemical features suggesting hyperthyroidism (with an increased free T4 and suppressed TSH) and that the more seriously ill among these women also have an elevation in serum free T3 (14). Some investigators showed that the severity of emesis correlated with the levels of free T4 and $\beta$-hCG (and TSH suppression) (15).

In this study however, there was no significant correlation between the severity of vomiting and the levels of FT3, FT4 , TSH and $\beta$-HCG. This lack of correlation may be explained by the fact that only a few of those studied (15.3\%) had hyperemesis gravidarum the rest having relatively mild emesis during pregnancy.

From this study it was shown that thyrotoxicosis doesoccur among women with emesis in pregnancy in this set-up. Screening protocol for it may be beneficial to such women.

\section{REFERENCES}

1. Glinoer, D. The regulation of thyroid function in pregnancy. Pathways of endocrine adaptation from physiology to pathology. Endocr. Rev. 1997; 18: 404-406.

2. Talbot, J.A., Lambert, A., Anobile, C. J.et al. The nature of human chorionic gonadotrophin glycoforms in gestational thyrotoxicosis. Clin. Endocr. 2001; 55: 33.

3. Utiger, R.D. Some women with hyperemesis gravidarum have transient hyperthyroidism. Clin. Thyroidology. 2002; 14: 56-58.

4. Vassart, G. and Dumont, J. E. The thyrotropin receptor and the regulation of thyroid function and growth. Endocr. Rev. 1992; 13: 596.

5. Hershman, J. M. Hyperthyroidism induced by trophoblastic thyrotropin. Mayo Clin. Proc. 1997; 47: 913.

6. Kimura, M. Gestational thyrotoxicosis and hyperemesis gravidarum. Clin. Endocr. 1993; 38: 345.

7. Yoshimura, M. and Hershman, J. M. Thyrotropic action of human chorionic gonadotropin. Thyroid Today. 1995; 5: 425.

8. Rodien, P., Bremont, C., Raffin, Sanson, M-L., et al. Familial gestational hyperthyroidism caused by a mutant thyrotropin receptor hypersensitive to human chorionic gonadotropin. New. Engl. J. Med. 1998; 339: 1823.

9. Goodwin, T. M., Montoro, M. and Mestman, J. H. The role of chorionic gonadotropin in transient hyperthyroidism of hyperemesis gravidarum. J. Clin. Endocr. Metab. 1992; 75:1333.

10. Abadi, A.L. Subclinical thyrotoxicosis. Postgraduate Med. J. 2001; 77: 29-31.

11. Anselmo, J., Kay, T., Dennis, K., et al. Resistance to thyroid hormone does not abrogate the transient thyrotoxicosis associated with gestation: report of a case. J. Clin. Endocr. Metab. 2001; 86: 4273.

12. Grun, J.P., Meuris, S., De Nayer, P.etal. The thyrotropic role of human chorionic gonadotropin (hCG) in the early stages of twin (versus single) pregnancy. Clin. Endocr. 1997; 46: 719.

13. Yeo, C.P., Hsu Chin, Khoo, D., Hsi Ko, Eng, P. et al. Prevalence of gestational thyrotoxicosis in Asian women evaluated in the 8th to 14 th weeks of pregnancy. Correlations with total and free beta human chorionic gonadotrophin. Clin. Endocr. 2001; 55: 391 .

14. Goodwin, T.M., Montoro, M. and Mestman, J.H. Transient hyperthyroidism and hyperemesis gravidarum: clinical aspects. Am. J. Obstet. Gynecol. 1992; 167: 648-651.

15. Goodwin, T.M. and Hershman, J.M.Hyperthyroidism due to inappropriate production of human chorionic gonadotropin. Clin. Obstet. Gynecol. 1997; 40: 32.

16. Patrice, R., Nicola, J. and Anne, L. et al. Abnormal stimulation of the thyrotrophin receptor during gestation. Human Reproduction Update. 2004; 10: 95-105. 